\title{
PAPER
}

\section{Increased blood-brain barrier permeability in type II diabetes demonstrated by gadolinium magnetic resonance imaging}

\author{
J M Starr, J Wardlaw, K Ferguson, A MacLullich, I J Deary, I Marshall
}

J Neurol Neurosurg Psychiatry 2003;74:70-76

See editorial commentary p6

See end of article for authors' affiliations

Correspondence to: Professor J M Wardlaw Department of Clinical Neurosciences, Western General Hospital NHS Trust, Bramwell Dott Building, Crewe Road, Edinburgh EH4 2XU, UK: jmw@skull.den.ed.ac.uk

Received

21 November 2001

In revised form 14 June

2002

Accepted 8 August 2002
Objectives: Patients with type II diabetes are at increased risk of cognitive impairment. The retinal and renal complications of diabetes follow microvascular damage permitting small arterioles to leak, hence the cerebral damage might also follow loss of blood-brain barrier (BBB) integrity. Magnetic resonance (MR) brain imaging with intravenous gadolinium (Gd) diethylenetriamine pentaacetic acid (Gd-DTPA) was used to identify increased BBB permeability.

Methods: Ten well controlled type II diabetic patients aged 65-70 years and 10 controls underwent MR brain imaging with fluid attenuated inversion recovery (FLAIR); T1 weighted (TIW) volumetric imaging before; and TIW volumetric imaging at 5, 15,30,45, 60, and 90 minutes after intravenous Gd-DTPA. The TIW image before Gd-DTPA was subtracted from the images at each time point after Gd-DTPA. Net signal intensity was plotted against time for different brain regions. White matter hyperintensities were scored from the FLAIR image.

Results: The signal intensity/time curves showed that brain signal intensity increased more in the diabetic group than controls during the first 15 minutes after Gd-DTPA, particularly in the basal ganglia $(p=0.018)$. Signal intensity in controls peaked at five minutes and diabetics at 15 minutes. Subjects with more white matter hyperintensities had greater signal increase after Gd-DTPA, whether diabetic or not $(p=0.001)$.

Conclusions: Increased BBB permeability with MR imaging was detected in patients with type II diabetes or white matter hyperintensities. Increased permeability of the BBB might account for some of the cerebral effects of type II diabetes, and so possibly also for the effect of other conditions that affect the microvasculature (like hypertension), on the brain.
$\mathrm{D}$ iabetes mellitus is associated with an increase in proliferative lesions in the small cerebral vessels. ${ }^{1}$ Functionally, the blood-retinal barrier is closely related to the blood-brain barrier $(\mathrm{BBB})^{2}$ and cortical capillaries in experimental models of diabetes exhibit similar microangiopathy to that found in the human retina in diabetes. ${ }^{34}$

A principal neuroradiological feature that may be associated with cerebral microvascular disease is "white matter hyperintensities" or "leukoaraiosis", a mixed condition of uncertain aetiology manifested on CT scans as hypodensity in the cerebral white matter, and as hyperintensities on T2, proton density or fluid attenuated inversion recovery (FLAIR) MR imaging. ${ }^{5}$ Recent longitudinal studies have highlighted diabetes as a risk factor for dementia, doubling the risk of senile dementia of the Alzheimer type. ${ }^{6}$ Several studies have established a correlation between the presence and extent of white matter hyperintensities and cognitive impairment, ranging from mild slowness of thinking to full blown dementia. $^{7}$ Thus diabetes mellitus could cause cognitive impairment by increasing cerebral microvascular disease. ${ }^{8}$

The cause of white matter hyperintensities is thought to be "ischaemic demyelination", " secondary to occlusion of small penetrating arteries. Perfusion studies have shown increased cerebral blood volume and hypometabolism in regions of white matter hyperintensities, ${ }^{10} 11$ and been interpreted as showing reduced cerebral blood flow. However, reduced cerebral blood flow and metabolism are consistent with hypocellularity (that is, neuronal loss). Whether the reduced cerebral blood flow caused the white matter hyperintensities (and if so what caused the reduced cerebral blood flow) is difficult to determine without longitudinal studies, as yet unavailable. The increased cerebral blood volume could also reflect a larger volume of distribution of the tracer used to measure perfusion if it did not remain intravascular but leaked across a damaged BBB.

Increased BBB permeability has been detected biochemically in both clinically diagnosed Alzheimer's disease ${ }^{12}$ and vascular dementia, but not previously using imaging. ${ }^{13}$ In a case report of a diabetic patient, Iwata and colleagues found that a region of the basal ganglia enhanced with gadoliniumDTPA (Gd-DTPA) before the onset of hyperglycaemic chorea, suggesting that increased BBB permeability preceded neuronal damage and the development of symptoms. ${ }^{14}$

We hypothesised that there might be increased BBB permeability in type II diabetes for several reasons: (a) the long observed leakage from small vessels in other organs (kidneys, eyes) with diabetes related damage; (b) diabetes increases cognitive impairment and is a risk factor for major causes of dementia in which it is possible that $\mathrm{BBB}$ permeability is increased $^{68}$; (c) there are limited biochemical data consistent with $\mathrm{BBB}$ leakage ${ }^{12}{ }^{13}$; and $(\mathrm{d})$ the single case report where such leakage was directly observed. ${ }^{14}$ We therefore hypothesised that type II diabetic patients would show increased BBB permeability associated with neuroimaging features suggesting cerebral small vessel disease, and that it would be possible to demonstrate this using MR brain imaging with intravenous Gd-DTPA.

Abbreviations: BBB, blood-brain barrier; Gd; gadolinium; FLAIR, fluid attenuated invasion recovery; TIW, TI weighted; MRI, magnetic resonance imaging; CSF, cerebrospinal fluid 

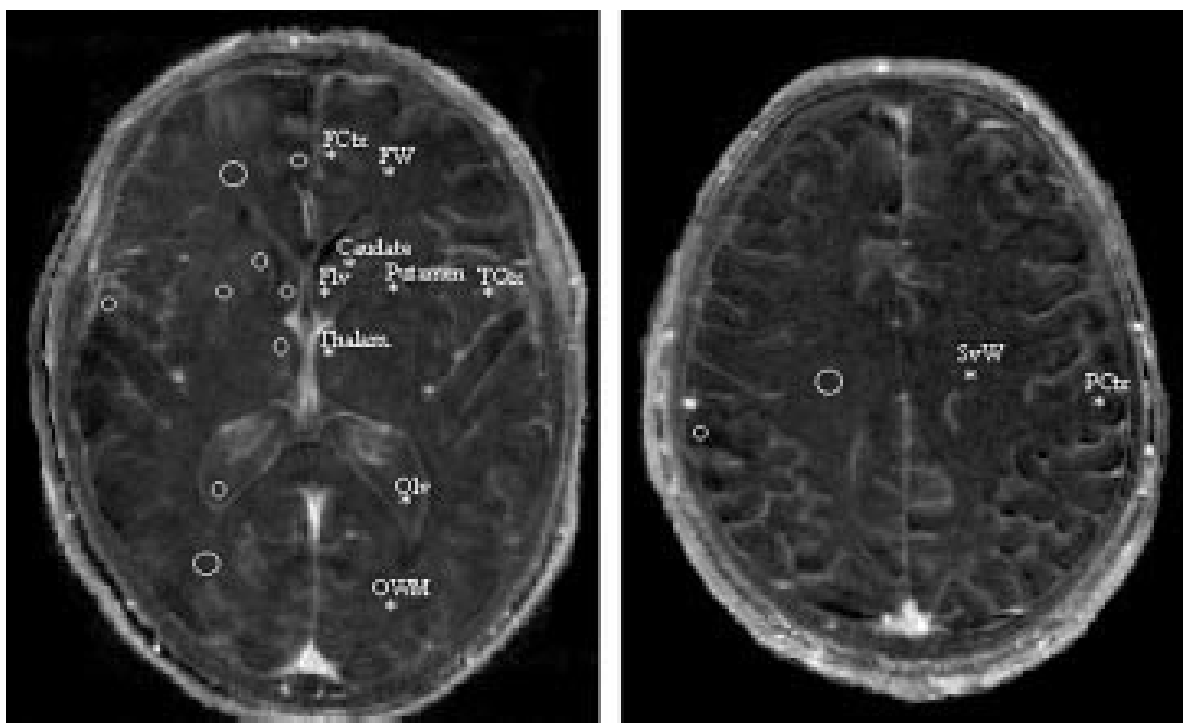

Figure 1 Regions of interest specified for signal intensity quantification on a "subtraction" image: Cortical areas (frontal, temporal, parietal), subcortical white matter areas (frontal, supraventricular, occipital), basal ganglia and thalamus (caudate, putamen, thalamus), and lateral ventricle (frontal, occipital). The terminal internal carotid arteries were also sampled (not shown).

\section{METHODS \\ Subjects}

Ten men aged 65 to 70 years with type II diabetes (NIDDM), well controlled by diet or oral hypoglycaemic drugs only, with no history of stroke, multiple sclerosis, cerebral tumour, or dementia were identified through our diabetic clinic. The subjects were not obese. All subjects gave written informed consent. Ten age matched, healthy men (control subjects) were selected from an established healthy cohort. These control subjects were all disease free, taking no medication with no past medical history of note, in particular of cerebral or cardiovascular disease or diabetes. There was no significant difference in the mean age of diabetic subjects (68.2 years), and controls (67.8 years) $(p=0.56)$. Four of the diabetic subjects were taking oral hypoglycaemic medication, four had a diagnosis of mild well controlled hypertension.

\section{MR procedures}

Each subject was positioned comfortably in the MR scanner (1.9T) lying supine with the head padded in the head coil to prevent movement, and instructed to keep still. An intravenous long line was inserted into an ante-cubital vein to avoid moving the subject during scanning. Symmetric head positioning was checked with a 3-view localiser before a FLAIR sequence was performed parallel to the AC-PC line (inversion recovery fast spin echo TR/TE 6000/2000/126, 5 mm slice width, no slice gap, field of view $24 \times 24 \mathrm{~cm}$, matrix $252 \times 256,24$ slices). Volumetric thin section axial T1 weighted images (TIW) of the whole brain were then obtained (TR/TE $29 / 9,3 \mathrm{~mm}$ slice width, no slice gap, field of view $=24 \times 24 \mathrm{~cm}$, matrix $240 \times 240$ ). Then an intravenous bolus injection of a gadolinium containing compound, gadopentetate dimeglumine diethylenetriaminepentaacetic acid (Gd-DTPA) ("Magnevist", Schering AG, Berlin, Germany) at double the normal dose for weight $(0.2 \mathrm{mmol} / \mathrm{kg})$ was given through the long line without moving the patient (followed by a saline flush). Thin section TIW volumetric imaging was repeated at 5,15 , 30, 45, 60, and 90 minutes after Gd-DTPA injection. Subjects remained in the scanner throughout listening to music and were instructed to keep still. To correct for any signal intensity drift during the long imaging time, we included a small bottle containing oil or water in the field of view (we had no reason to suspect this, but merely wished to be cautious).

\section{Image analysis}

MR data were transferred to a Sun workstation (Sun Micro Systems, Mountain View, CA) and processed offline. The TIW volumetric images prior to Gd-DTPA injection were aligned with and subtracted from the TIW volumetric images obtained at each of the time points $(5,15,30,60$, and 90 minutes) after Gd-DTPA injection using Statistical Parametric Mapping (SPM 95, Friston, FIL, London). Thus the subtraction images consisted only of the difference in signal intensity attributable to the Gd-DTPA at each time point. The ANALYZE software package (Mayo Clinic, Rochester, version 7.5), was then used to assess signal intensity change in prespecified regions of interest (see below and fig 1). Signal intensity drift during imaging was assessed using data from the phantoms. We used a mathematical correction derived from the phantom data to adjust for any scanner signal intensity drift. Although the signal intensity drift was minimal, we excluded from the analysis the three subjects (all diabetics) who did not have phantom data so as to be certain that any signal intensity change in the brain was attributable only to the presence of Gd-DTPA. We assumed that the signal intensity change from baseline was directly proportional to the amount of Gd-DTPA in the region of interest at each imaging time point, because the effect that the presence of the Gd-DTPA had on the magnetic properties of the tissue. Mean signal intensities in the left and right hemispheres were measured from the subtraction images at each time point for the following regions of interest: terminal internal carotid arteries (ICA), cortical areas (frontal, temporal, parietal), subcortical white matter areas (frontal, parietal, occipital), basal ganglia (caudate, putamen and thalamus), lateral ventricles (frontal and occipital horns) (fig 1). All image analyses were performed blind to the subject's clinical status and the results of any other imaging.

\section{White matter hyperintensities scores}

The following scales were used to score the white matter hyperintensities on the FLAIR images, blind to clinical status and other imaging results by a neuroradiologist: Breteler, ${ }^{15}$ Longstreth ${ }^{16}$ van Swieten, ${ }^{17}$ and Fazekas. ${ }^{18}$ Atrophy was scored as either present or absent and whether deep (that is, mainly resulting in ventricular enlargement) or superficial (that is, mainly affecting the cortex and causing sulcal enlargement) structures were affected more than one would reasonably 
Table 1 Mean scores on white matter hyperintensities scales for 10 diabetic men aged $65-70$ years and 10 age matched normal men

\begin{tabular}{|c|c|c|}
\hline Score method & $\begin{array}{l}\text { Control } \\
\text { subjects }\end{array}$ & $\begin{array}{l}\text { Diabetic } \\
\text { subjects }\end{array}$ \\
\hline Breteler $^{16}$ & 0.9 & 1.4 \\
\hline Longstreth $^{17}$ & 2.8 & 3.1 \\
\hline van Swieten $A^{18}$ & 0.9 & 1.0 \\
\hline van Swieten $\mathrm{P}^{18}$ & 1.0 & 1.1 \\
\hline Fazekas $\mathrm{PVH}^{19}$ & 1.8 & 2.1 \\
\hline Fazekas DWMH ${ }^{19}$ & 1.3 & 1.4 \\
\hline
\end{tabular}

$\mathrm{PVH}$, periventricular hyperintensities; $\mathrm{DWMH}$ diffuse white matter hyperintensities.

expect for someone of their age, using a simple subjective scale devised "in house".

\section{Statistical analysis}

We used analysis of variance and covariance to develop models to describe the pattern of signal intensity change over time. In these models, key variables included side (left or right), depth of brain region (cortical, subcortical/white matter, basal ganglia, cerebrospinal fluid (CSF), and carotid arteries) and anterior to posterior position (as subjects were lying supine over a prolonged period), and adjusting for the small sample size. ${ }^{19}$ We investigated the change in MR signal intensity over time using a repeated measures design and polynomial contrasts for time effects. Time trends are thus described as linear if the pattern of change is consistent with an overall
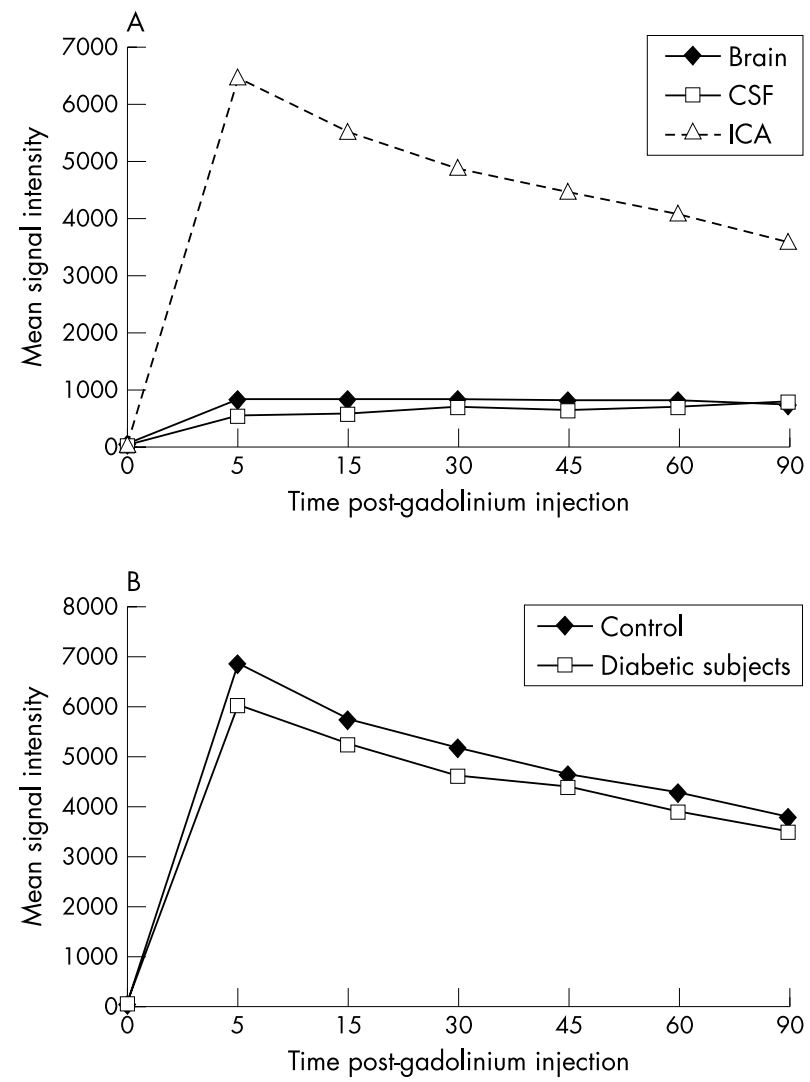

Figure 2 (A) Change in signal intensity for all patients averaged, in the terminal internal carotid artery (ICA), brain parenchyma (all regions averaged) and cerebrospinal fluid (CSF-all regions arranged) with time after injection. (B) Change in signal intensity in blood (measured from the ICA) in diabetic patients compared with controls. linear increase or decrease over time, quadratic if there is an initial increase followed by a subsequent but less rapid increase (or vice versa), or cubic if change over time is consistent with a third order function. Previous kinetic studies suggested that in the absence of BBB leakage, an exponential fall in signal intensity would be observed, ${ }^{20}$ consistent with a linear time trend and accounting for the increasing time interval spacing of our observations. A quadratic time trend with an initial rise followed by a fall in intensity would be detected if BBB leakage were present. In this analysis, we assumed that signal intensity was directly related to the amount of Gd-DTPA in the region of interest.

\section{RESULTS}

There were no significant differences in white matter lesion scores between the diabetic and control subjects. Four subjects had mainly cortical atrophy and eight had atrophy mainly affecting the deep structures causing enlargement of the ventricles. These abnormalities were absolutely evenly distributed between the diabetics and controls (table 1).

\section{Signal change after Gd-DTPA injection}

Signal change in blood after Gd-DTPA injection

In the left and right internal carotid arteries, signal intensity after Gd-DTPA injection peaked at five minutes after injection then declined steadily over time to 90 minutes $(p<0.001)$. At 90 minutes, the signal was much less than it had been at five minutes after Gd-DTPA injection (fig 2A). The decline in signal intensity was significantly more rapid in the internal carotid arteries than in all brain regions $(\mathrm{p}<0.001)$ (fig $2 \mathrm{~A})$. There was no significant difference in signal intensity change in the internal carotid arteries between diabetics and controls (fig 2B).

\section{Signal intensity change in CSF after Gd-DTPA injection}

There was a significant and progressive increase in signal intensity after Gd-DTPA injection in the CSF $(F=5.21$, $\mathrm{p}=0.001$, fig 3) throughout the whole imaging period. In addition, the signal was significantly higher in occipital horns than in frontal horns throughout the 90 minutes $(F=4.80$, $p=0.044)$. The linear time trend of the slope of the signal increase was significant $(F=12.22, \mathrm{p}=0.003)$, and the cubic time trend of the slope was also marginally significant $(F=4.52, \mathrm{p}=0.049)$ as shown in fig 3 , suggesting two phases to

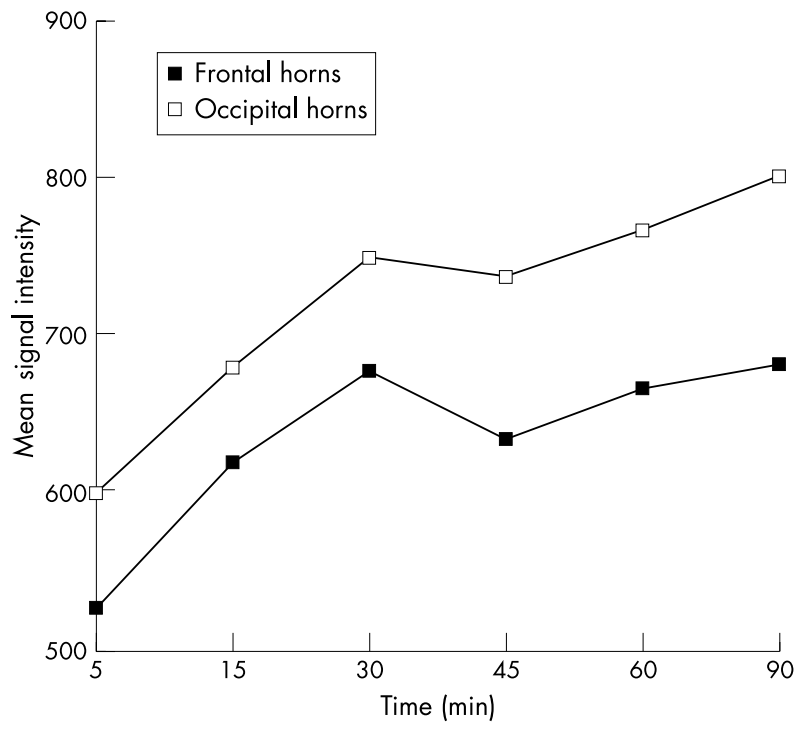

Figure 3 Gd-DTPA signal intensity from CSF in frontal and occipital horns in seven male diabetic and 10 healthy subjects aged $65-70$ years. 
the accumulation of Gd-DTPA in the CSF. There was no difference in the shape of the signal intensity curve in the CSF between diabetic subjects and healthy controls over time $(p=0.83)$, nor between subjects with or without predominantly cortical $(p=0.43)$ or central cerebral atrophy $(p=0.78)$. However, subjects with more white matter hyperintensities (Longstreth score 4 to 8 ) had greater increase in signal intensity in the CSF after Gd-DTPA over time $(F=3.07, \mathrm{p}=0.019)$ than those with less white matter hyperintensities (Longstreth score 0 to 3), regardless of whether they were diabetic or not. Similar effects were seen for van Swieten A and Fazekas white matter hyperintensities scores.

\section{Signal intensity change in the brain parenchyma after Gd-DTPA}

For diabetic and control subjects considered together, there was a greater rise in signal intensity immediately after Gd-DTPA injection in deep (basal ganglia) than in superficial (cortex) structures $(p=0.045)$ (fig 4$)$. There was also a suggestion of differences between different regions of the brain but this trend did not reach statistical significance $(\mathrm{p}=0.068)$.

Comparing diabetics with controls, the overall signal intensity was significantly greater in the basal ganglia region of diabetics than of controls throughout the 90 minutes after Gd-DTPA injection (fig 4). Also the pattern of signal intensity change differed between diabetics and controls. In control subjects, the signal intensity was highest in the brain parenchyma at five minutes after injection (all regions) and declined with time thereafter $(p=0.067)$, in a linear pattern (linear time trend $p=0.028$ ). However, in diabetic subjects, the time plot for all brain regions indicated that signal intensity after Gd-DTPA injection continued to increase between five and 15 minutes before starting to decline. The difference between diabetics and controls was most marked in the basal ganglia. From 30 minutes after injection onwards, the time trends were similar for diabetic subjects and controls-that is, a steady decline in signal intensity. Thus signal intensity continued to rise to an observed maximum at 15 minutes after Gd-DTPA injection in diabetic subjects, whereas control subjects showed a steady linear decline in signal intensity from five minutes after injection onwards.

Signal intensity change in the brain parenchyma after Gd-DTPA injection: association with white matter hyperintensities

Taking diabetic and control subjects together, those with higher white matter lesion scores had greater signal intensity increase at 15 minutes than at five minutes after injection (Longstreth score, ${ }^{16} \mathrm{p}=0.009$ ) and lower signal intensity at 90 minutes than at 60 minutes (Longstreth score, $p=0.009$ ). Similar significant effects were found for white matter hyperintensities classified by the Fazekas periventricular hyperintensities $(\mathrm{PVH})$ score $^{18}(\mathrm{p}=0.023)$ and the Fazekas diffuse white matter hyperintensities (DWMH) score $(p=0.005)$. The sample size was too small to test whether the pattern of signal change in the white matter was associated with the severity of white matter disease.

Signal intensity change after $\mathrm{Gd}$ injection in frontal and occipital brain parenchyma and CSF

Signal intensities after Gd-DTPA injection were significantly higher in brain than in CSF over the 90 minutes of imaging $(\mathrm{p}=0.019)$. The signal intensities were also significantly higher in the occipital brain and the CSF in the occipital horns of the lateral ventricles, than in frontal brain and CSF in the frontal horns $(p=0.002)$. There was no significant difference between left and right sides $(p=0.15)$. There was also a difference between the pattern of signal intensity change in brain and that in CSF over time $(p=0.001)$. Presence or absence of
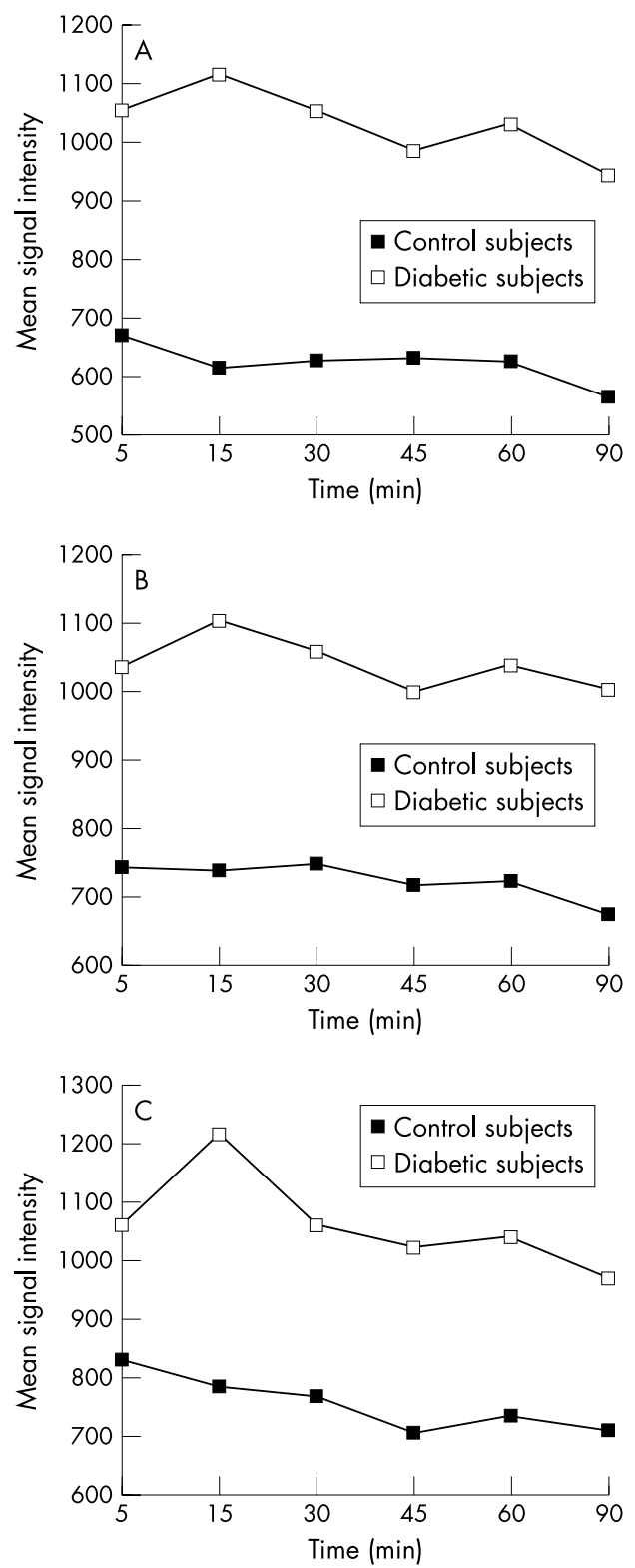

Figure 4 Gd-DTPA signal intensity from (A) cortical, (B) subcortical, and (C) basal ganglia brain regions in diabetic patients and healthy controls aged 65-70 years.

diabetes, sulcal atrophy, or ventricular atrophy were not related to the difference in time course of signal intensity change between brain and CSF, but patients with greater white matter disease scores had more pronounced differences between frontal and occipital horns and in the pattern of signal intensity change over time in CSF.

\section{DISCUSSION}

In this small and comparatively preliminary study, we have demonstrated features consistent with increased BBB permeability in diabetic patients compared with controls, and in those with more white matter hyperintensities than in those with fewer. This simple technique is worthy of further development for use in studies of the effects of "vascular" disease on the brain and of mechanisms of dementia.

The differences we have observed between diabetic patients and controls are unlikely to be attributable to poor renal function in the diabetic patients, or greater obesity among the diabetic patients. These were well controlled maturity onset 
diabetes with normal renal function and were not obese. Furthermore, there was no difference in the time course of signal intensity change in the internal carotid artery (that is, the main blood pool) between diabetics and controls. If our observations were attributable to prolonged retention of Gd-DTPA in the blood because of renal impairment, we should have seen a plateau in signal intensity in the blood from five minutes or a slower decline in signal intensity in the blood in diabetics than in controls and we did not (fig 2). Hypertension is a risk factor for cerebrovascular disease. We did not "adjust" for this in our analysis as the sample size was too small, and the four diabetics with hypertension had only well controlled mild hypertension. There was no evidence on examination of individual cases that the diabetics with hypertension had a different pattern of signal change compared with the diabetics without hypertension or the controls, but the sample size was too small to examine this properly.

\section{Baseline and time adjusted observations}

There were no differences between the diabetic and healthy control subjects in the range of white matter hyperintensities as quantified by several rating scales or in the atrophy. This was as expected with the small sample size. There was significant heterogeneity in signal intensity after Gd-DTPA injection between different brain regions. Signal intensity after Gd-DTPA injection was greatest in deeper hemisphere brain structures - that is, signal intensity was significantly higher in the basal ganglia than in more superficial cortical regions. This could relate to either greater cerebral perfusion brain of these deeper structures (if we were simply detecting intravascular Gd-DTPA) or increased BBB permeability in the basal ganglia. Secondly, there was a significant intensity gradient increasing from frontal to occipital brain regions. This anteroposterior gradient was also observed in the CSF. Subjects lay supine in the scanner for about two hours to acquire scans before and up to 90 minutes after injection of Gd-DTPA. It is possible that the gradient reflects gravitational effects on "cerebral haemodynamics" over this prolonged period in one position. In the CSF, it might also reflect the greater production of CSF in the occipital horns by the choroid plexus.

\section{Effects of diabetes}

There was a trend for signal intensity after Gd-DTPA injection to peak at 15 minutes and then fall over time, and within this trend, the linear decline was significant. Given the time spacing between observations (10 minutes, 15 minutes, 15 minutes, 30 minutes, 30 minutes) the statistical linear decline is closer to a real time exponential decline consistent with first order kinetics of Gd-DTPA removal from the brain as a whole. ${ }^{20}$ However, the diabetic subjects did not show such a simple exponential decline attributable to a significant increase in signal intensity over the first 15 minutes after injection. The significant differences detected between diabetic and control subjects for signal intensity change over the first 30 minutes after Gd-DTPA injection (as statistically detected by the quadratic time trend) are consistent with increased BBB permeability. The signal intensity increase seen in the diabetic subjects is consistent with previous theoretical models and similar to that observed in single lesions in multiple sclerosis. ${ }^{20}$ The detection of the greatest difference between diabetic and control subjects in caudate, putamen, and thalamus is consistent with the greater blood flow per unit volume of tissue in these regions and with known pathological observations of $\mathrm{BBB}$ breakdown. ${ }^{21}$ After 30 minutes, the time profile for diabetic subjects was similar to that of the healthy controls, again consistent with first order kinetics.

\section{Effects of white matter hyperintensities}

Subjects with more white matter hyperintensities had a peak in signal intensity at 15 minutes after Gd-DTPA injection, a more rapid decline in signal intensity thereafter, and more rapid increase in signal in CSF than subjects with fewer white matter hyperintensities. These relations were consistently related to the amount of white matter disease as quantified by several different scores. Subjects with more white matter hyperintensities had a significant increase in signal intensity after Gd injection between five and 15 minutes, but a greater decrease by 90 minutes. This finding was similar, although with a longer wash out phase, to the increased BBB permeability detected for the diabetic (versus control) subjects. We would hypothesise a common mechanism because it would be consistent with the evidence that people with diabetes are more likely to have white matter hyperintensities. ${ }^{22}$ Moreover, the quadratic time trend (increase then decrease) was seen not only for those white matter scores with an emphasis on periventricular changes (Longstreth and Fazekas periventricular lesions), but also for Fazekas deep white matter score that reflects changes remote from the ventricles. Hence the longer period over which signal intensity rises and falls may indicate that these lesions represent lacunar "reservoirs" within the brain that exchange with plasma.

\section{Changes in the CSF}

Examination of the CSF data combined with that from the brain reveals complex kinetics in which white matter hyperintensities, or whatever pathophysiological process they are a surrogate for, seem to play a part. The increase in Gd signal intensity in CSF over time could reflect flow at three places: the choroid plexus, brain capillaries, and transudation from the brain extracellular, extravascular tissue space. The linear time trend increase is likely to reflect flow from blood, probably largely at the choroid plexus, as with the time spacing this would reflect the observed exponential decline in blood Gd-DTPA concentration. Presumably small vessels in the choroid plexus could be affected by whatever process affects the small vessels in the brain parenchyma to increase permeability. In addition there is a quadratic time trend associated with increased white matter hyperintensities. This trend is likely to relate to flow at places other than the choroid plexus and thus, by implication, reflect increased BBB permeability. The dip in signal around 45 minutes would be more consistent with brain tissue acting as a buffer for Gd-DTPA that has crossed the BBB soon after injection and is flowing back both into brain capillaries and directly into CSF over time. The observation that frontal and occipital horn CSF signal intensity rises to approach the falling intensities in the respective adjacent brain regions would support this hypothesis.

Thus, while our interpretation of the data are somewhat speculative, theoretical models of BBB permeability may need to consider interchange with CSF in addition to those between blood and brain. ${ }^{20}$ Moreover, such models could helpfully consider the heterogeneity between regions we observed both in diabetic and healthy control subjects.

\section{Relation with previous studies}

Our in vivo data suggesting increased BBB permeability are consistent with several animal and human postmortem studies. $^{349}$ Skoog and colleagues ${ }^{23}$ measured CSF/blood albumin levels as an index of BBB permeability and found an increase at an early stage in older people who were later clinically diagnosed as having vascular dementia or Alzheimer's disease. Stroke prone hypertensive rats, frequently used as a model for human cerebrovascular disease, are susceptible to $\mathrm{BBB}$ breakdown ${ }^{24}$ suggesting a close relation between cerebral macrovascular and microvascular disease. Such a relation may be particularly relevant to lacunar infarcts traditionally associated with hypertension. We recently described a variant, or possibly intermediary stage, of lacunar 
infarction (type Ib, or "incomplete lacunar infarction") ${ }^{21}$ as selective neuronal necrosis with relative preservation of glial elements around a penetrating artery, which was essentially the same as the "oedema related gliosis" described by Ma and Olsson. ${ }^{26}$ The striking histological similarities to oedema related lesions induced in experimental animals ${ }^{25}$ parenchymal vacuolation, perivascular lesion distribution, and fibrinogen immunoreactivity-suggest an important role for increased permeability leading to oedema mediated tissue damage, whether or not associated with permanent vessel occlusion. Furthermore, such features had been observed previously, but their relevance to ischaemic small vessel disease overlooked possibly because of the observed patency of the associated small arteries. ${ }^{21}$ The significant associations between signal intensity change after Gd-DTPA injection, diabetes, and white matter hyperintensities we observed in vivo would fit very well with pathological processes entailing increased BBB permeability and perivascular oedema. Similar $\mathrm{BBB}$ breakdown is observed in rats with chronic renal hypertension, and reduced by treatment with the angiotensin converting enzyme inhibitor enalapril. ${ }^{28}$ This latter observation suggests that the BBB may be a potential treatment target for neurovascular protection. The methods we describe in this study could be used to quantify any treatment effects.

There is one previous study investigating BBB permeability in humans with white matter hyperintensities using Gd-DTPA and sequential MR imaging. This study of 10 patients with dementia failed to find evidence of Gd-DTPA leak into the brain on MR, but this failure could be methodological. ${ }^{29}$ The sample size was small (about half of ours), a less detailed MR imaging sequence was used, and the image before the Gd-DTPA injection was not subtracted from the images after Gd-DTPA injection to determine absolute change in signal. In contrast, another study in 18 elderly dogs, imaged up to 12 minutes after Gd-DTPA injection, did find contrast leak in the one dog with white matter hyperintensities. ${ }^{30}$

\section{Methodological issues}

The sample we studied was restricted in age (65 to 70 years) and sex (male only). We also only included subjects with well controlled type II diabetes to avoid possible confounding attributable to previous hypoglycaemic episodes. The sample size was too small to demonstrate reliably any relation with cognition. ${ }^{8}$ These observations therefore need repeating in a broader range of subjects, with adjustment for comorbidity such as hypertension. We excluded data from three subjects without phantom data because the signal intensity values after Gd-DTPA injection seemed extreme and we could not be certain, at this stage in the work, that this was not attributable to drift in inherent scanner intensity. Future studies with larger sample sizes should help to provide more data on the breadth of change in signal intensity to be expected, but at this stage we wished to be cautious.

We did not measure absolute $\mathrm{T} 2$, and there is evidence that measured signal change after Gd-DTPA in experimental studies is influenced by T2 signal intensity. However, the magnitude of those effects is small and very unlikely to account for the differences, or highly consistent patterns that we have observed between different brain and CSF regions over time. However, future studies should probably correct for this to be absolutely methodologically precise.

\section{Implications for future research}

The findings from this study require replication in other subjects with diabetes and with white matter hyperintensities and various degrees of cognitive impairment. As the significant changes over time were seen comparatively early in diabetes, more frequent measurement of signal intensity after Gd-DTPA injection over the first 30 minutes may increase power to detect differences in BBB permeability. Furthermore, it would not be necessary to continue imaging for as long as 90 minutes-30 minutes after injection should suffice. The method could also be used to quantify BBB permeability in other diseases. The complex kinetics of Gd-DTPA within the brain and the CSF suggest that future models should include separate compartments for blood, brain, and CSF, and allow for bi-directional flow. Should future studies confirm BBB permeability as a correlate of brain pathology, the ability to quantify BBB permeability in vivo will facilitate evaluation of targeted interventions.

\section{ACKNOWLEDGEMENTS}

We thank Dr J McKnight for providing access to diabetic clinic patients. This work was directly supported by grants from Diabetes UK and the Scottish Higher Education Funding Council (SHEFC). The image acquisition and analysis were performed in the SHEFC Brain Imaging Research Centre for Scotland (www.dcn.ed.ac.uk/bic), which was established with a JREI grant from the Medical Research Council, SHEFC, and industrial support from GE Medical Systems, Boehringer Ingelheim, Novartis and Schering.

Authors' affiliations

J M Starr, K Ferguson, A MacLullich, I J Deary, SHEFC Centre for the Study of the Ageing Brain, University of Edinburgh, Western General Hospital, Edinburgh, UK

J Wardlaw, Department of Clinical Neurosciences, University of Edinburgh, Western General Hospital

I Marshall, Department of Medical Physics, University of Edinburgh, Western General Hospita

Competing interests: none declared.

\section{REFERENCES}

1 Gorelick PB, Sacco RL, Smith DB, et al. Prevention of a first stroke: a review of guidelines and a multidisciplinary consensus statement from the National Stroke Association. JAMA 1999;281:11112-20.

2 Alex M, Baron EK, Goldenberg S, et al. An autopsy study of cerebrovascular accidents in diabetes mellitus. Circulation 1962;25:663-73

3 Taarnhoj J, Alm A. The effect of diabetes on transport through the blood-retinal and blood-brain barrier in rats. Graefes Arch Clin Exp Ophthalmol 1991;229:291-3.

4 Junker V, Jaggi C, Bestetti $G$, et al. Basement membrane of hypothalamus and cortex capillaries from normotensive and spontaneous hypertensive rats with streptozotocin-induced diabetes. Acta Neuropathol 1985;65:202-8.

5 Hachinski VC, Potter $\mathrm{P}$, Merskey $\mathrm{H}$. Leukoaraiosis: an ancient term for a new problem. Can J Neuro Sci 1986;13:533-4.

6 Ott A, Stolk RP, van Harskamp F, et al. Diabetes mellitus and the risk of dementia. The Rotterdam Study. Neurology 1999;53:1937.

7 Van Gijn J. Leukoaraiosis. In: Warlow CP, Dennis MS, van Gijn J, et al, eds. Stroke: a practical guide to management. 2nd edn. Oxford: Blackwell Scientific, 2001:207-11.

8 Strachan MW, Deary IJ, Ewing FM, et al. Is type II diabetes associated with an increased risk of cognitive dysfunction? A critical review of published studies. Diabetes Care 1997;20:438-45.

9 Zhang WW, Ollson Y. The angiopathy of subcortical arteriosclerotic encephalopathy. Acta Neuropathol (Berlin) 1997;93:219-24.

10 Yao H, Sadoshima S, Ibayashi S, et al. Leukoaraiosis and dementia in hypertensive patients. Stroke 1992;23:1673-7.

11 Markus HS, Lythgoe DJ, Ostergaard L, et al. Reduced cerebral blood flow in white matter in ischaemic leukoaraiosis demonstrated using quantitative exogenous contrast based perfusion MRI. J Neurol Neurosurg Psychiatry 2000;69:48-53.

12 Blennow K, Wallin A, Fredman P, et al. Blood-brain barrier disturbance in patients with Alzheimer's disease is related to vascular factors. Acta Neurol Scand 1990:81:323-6.

13 Wallin A, Blennow K, Fredman P, et al. Blood brain barrier function in vascular dementia. Acta Neurol Scand 1990;81:318-22.

14 Iwata A, Koike F, Arasaki K, et al. Blood brain barrier destruction in hyperglycemic chorea in a patient with poorly controlled diabetes. $J$ Neurol Sci 1999:163:90-3.

15 Breteler MMB, van Amerongen NM, van Swieten JC, et al. Cognitive correlates of ventricular enlargement and cerebral white matter lesions on magnetic resonance imaging. The Rotterdam study. Stroke 1994:25: 1 109-15.

16 Longstreth WT, Manolio TA, Arnold A, et al. Clinical correlates of white matter findings on cranial magnetic resonance imaging of 3301 elderly people. The Cardiovascular Health study. Stroke 1996;27:1274-282.

17 van Swieten JC, Hiidra A, Koudstaal PJ, van Giin J. Grading white matter lesionson CT and MRI: a simple scale. J Neurol Neurosurg Psychiatry 1990;53:1080-3. 
18 Fazekas F, Chawluk JB, Alavi $A$, et al. MR signal abnormalities at 1.5T in Alzheimer's dementia and normal aging. AJNR 1987;8:421-6.

19 Huynh H, Feldt LS. Estimation of the Box correction for degrees of freedom from sample data in the randomized block and split-block designs. J Educational Statistics 1976;1:69-82.

20 Tofts PS. Modeling tracer kinetics in dynamic Gd-DTPA MR imaging. J Magn Reson Imaging 1997;7:91-101.

21 Lammie GA, Brannan F, Wardlaw JM. Incomplete lacunar infarction (Type 1b lacunes). Acta Neuropathol 1998;96:163-7.

22 Araki Y, Nomura M, Tanaka H, et al. MRI of the brain in diabetes mellitus. Neuroradiology 1994;36:101-3.

23 Skoog I, Wallin A, Fredman P, et al. A population study on blood-brain barrier function in 85-year-olds: relation to Alzheimer's disease and vascular dementia. Neurology 1998:50:966-71.

24 Absher PM, Hendley E, Jaworski DM, et al. Impairment of the blood-brain barrier: a potential surrogate delineating the determinants of cerebral bleeding caused by fibrinolytic drugs. Coron Artery Dis $1999 ; 10: 413-20$.
25 Yamagata K, Tagami M, Nara Y, et al. Faulty induction of blood-brain barrier functions by astrocytes isolated from stroke-prone spontaneously hypertensive rats. Clin Exp Pharmacol Physiol 1997;24:686-91.

$26 \mathrm{Ma} \mathrm{K}-\mathrm{C}$, Olsson Y. Structural and vascular permeability abnormalities associated with lacunes of the human brain. Acta Neurol Scand 1993;88:100-7.

27 Fredriksson K, Kalimo H, Nordborg C, et al. Cyst formation and glial response in the brain lesions of stroke-prone spontaneously hypertensive rats. Acta Neuropathol 1988;76:441-50.

28 Nag S, Kilty DW. Cerebrovascular changes in chronic hypertension. Protective effects of enalapril in rats. Stroke 1997;28:1028-34.

29 Wahlund LO, Bronge L. Contrast-enhanced MRI of white matter lesions in patients with blood-brain barrier dysfunction. Ann NY Acad Sci 2000;903:477-81.

30 Su $M-Y$, Head E, Brooks WM, et al. MRI of anatomic and vascular characteristics in a canine model of human ageing. Neurobiol Ageing $1998 ; 19: 479-85$.

\section{HISTORICAL NOTE}

\section{Willis on narcolepsy}

$\mathrm{N}$

arcolepsy, a syndrome of unknown origin is characterised by excessive daytime sleepiness and often disturbed nocturnal sleep and pathological manifestations of REM sleep. From the Greek v $\alpha \rho \kappa \omega \sigma \tau \varsigma$ meaning to benumb or stupefy, narcolepsy is often called Gélineau's syndrome, after the French physician Jean Baptiste Edouard Gélineau, who in 1880 gave the first comprehensive description. ${ }^{1}$ Westphal described it but more notable among early recognisable clinical portrayals is that of Thomas Willis. Willis did not use the word narcolepsy that was first coined by Gélineau as:

"a rare neurosis ... characterised by an urgent necessity to sleep, sudden and of short duration which recurred at intervals more or less long."

Willis noted that it was not just an embarrassing somnolence but a humoral disease. $\mathrm{He}$ thought that the body was able to produce its own narcotic substances.

" . . . a sleepy disposition -they eat and drink well, go abroad, take care well enough of their domestick affairs, yet whilst talking or walking, or eating, yea their mouthes being full of meat, they shall nod, and unless roused by others, fall fast asleep. ${ }^{\prime 34}$

Adie and later Daniels recognised an association between daytime sleepiness, cataplexy, sleep paralysis, and hypnagogic hallucination-associations not known to Willis or Gélineau.

Binns in 1842 is usually credited ${ }^{6}$ with the first report of paralysis which occurred in a daytime nap": hence his term "daymares":

"utter incapacity for motion or speech, difficult respirations, and extreme dread."

More recently Mignot and others have shown that neurons that secrete hypocretins (primarily in the hypothalamus) are depleted or damaged in narcolepsy. ${ }^{8}$ And, they are absent or diminished in CSF of most narcoleptics studied. Although dogs have a mutation of the gene encoding hypocretins (orexins), so far consistent genetic anomalies have been rare in man.

J M S Pearce

304 Beverley Road, Anlaby, Hull HU10 7BG, UK imspearce@freenet.co.uk

\section{References}

1 Gélineau J. De la narcolepsie. Gaz Hôp Paris 1880;53:626-8, 635-7.

2 Compston A. Thomas Willis (1621-1675). Paper read at the meeting of the Association of British Neurologists, Exeter, 6 April 2000.

3 Lennox WC. Thomas Willis on narcolepsy. Archives of Neurology and Psychiatry 1939:41:348-51.

4 Dewhurst K. Thomas Willis and the foundation of British neurology. In: Rose FC Bynum WF, eds. Historical aspects of the neurosciences. New York: Raven Press, 1982:327-46.

5 Adie WJ. Idiopathic narcolepsy: a disease sui generis: with remarks on the mechanism of sleep. Brain 1926;49:275-306.

6 Pearce JMS. Early descriptions of sleep paralysis. J Neurol Neurosurg Psychiatry 1993:56:1302.

7 Binns E. The anatomy of sleep; or, the art of procuring a sound and refreshing slumber at will. London: J Churchill, 1842. cited by Bell CC et al. J Natl Med Assoc 1984;76:501-8.

8 Travis J. Brain-cell loss found in narcolepsy. Science News 2000 Sept 2. 